\title{
Cognitive Defusion for Psychological Distress, Dysphoria, and Low Self-Esteem: A Randomized Technique Evaluation Trial of Vocalizing Strategies
}

\author{
Marchion J. Hinton \& Scott T. Gaynor
}

\begin{abstract}
Cognitive defusion procedures, as used in acceptance and commitment therapy (ACT), attempt to alter how an individual relates to negative thoughts (without challenging, disputing, or trying to change their content) so as to promote psychological flexibility, the key feature of the ACT model of adaptive functioning. The current study examined the efficacy of three, once weekly, sessions of cognitive defusion (CD) compared to a concurrent waitlist (WL) condition for university students reporting elevated distress, dysphoria, and low self-esteem. Participants $(N=22)$ were randomized to $\mathrm{CD}$ or WL, with the latter offered CD following their WL participation. The combined data from all those offered CD were also compared to a supportive therapy benchmark established from archival data. Intent-to-treat analyses showed large changes in distress, depressive symptoms, self-esteem, defused awareness of thoughts, psychological flexibility, and automatic thoughts favoring CD over WL. These effects were replicated when the WL group then received $\mathrm{CD}$ and persisted (or further improved) at 1-month follow-up. CD within-group effect sizes were also reliably larger than those derived from a comparable supportive therapy condition. That CD was associated with significant changes in both outcome and process variables is consistent with the existing literature and with the view that cognitive defusion techniques (particularly vocalizing strategies) represent an active component of ACT. Future research employing more stringent concurrent controls, clinical samples, and multi method assessments that allow for temporally sequenced tests of mediators is needed to more fully substantiate the effects of cognitive defusion.

Keywords: vocalizing, cognitive defusion, acceptance and commitment therapy
\end{abstract}

Acceptance and commitment therapy (ACT, Hayes, Strosahl, \& Wilson, 1999) is one of several behavior therapies that place a significant emphasis on acceptance and mindfulness practices (Hayes, 2004; Hayes, Follette, \& Linehan, 2004). Acceptance and mindfulness practices in ACT involve "the conscious abandonment of a direct change agenda in the key domains of private events, self, and history, and an openness to experiencing thoughts and emotions as they are, not as they say they are" (Hayes \& Pankey, 2003, p. 5). ACT is based on an articulated model of psychotherapy wherein mindfulness- and acceptance-based practices and behavior change strategies coalesce to enhance psychological flexibility (Hayes, Luoma, Bond, Masuda, Lillis, 2006). There have been a number of promising clinical trials suggesting the efficacy of ACT, that indices of psychological flexibility change with treatment, and, in some cases, that these changes in flexibility served as a mediator of outcome (Hayes et al., 2006). Such data support links between multi-component ACT packages, psychological flexibility, and outcome, but do not speak to what aspects of ACT were influential. Thus, the value of the various treatment components in the ACT model are left open to question, with the role of the acceptance-based practices often representing a point of focus in the debate (Arch \& Craske, 2008).

With respect to negative thoughts, ACT emphasizes cognitive defusion (Hayes, 2004). In cognitive defusion no attempt is made to change the content or frequency of negative thoughts. Instead, 
the focus is on changing how the individual relates to his/her thoughts, such that thoughts are not taken literally, as statements of truth or fact, but rather simply represent verbal activity. For instance, if "I'm a loser" is taken literally it will likely have undesirable functions, such as eliciting emotional discomfort, decreasing motivation to engage in approach behavior and promoting avoidance behavior (e.g., "Why call anyone, no one wants to hang out with a loser"), and occasion other negative self-talk that further emphasizes the importance of cognitive content (e.g., "Only real losers have the thought 'I'm a loser'") or validates belief in the original cognitive content (e.g., "Here I am sitting home on a Friday night, what a loser"). However, if "I'm a loser" can be experienced as what it is (i.e., a thought) and not what it is says it is (a statement of fact about the self) the unhelpful functions of the thought may be reduced without any attempt to alter the frequency or content. It is not that the individual no longer has the thought, but when it occurs its negative inhibitory functions are reduced, promoting increased psychological flexibility, the general goal of all the ACT strategies (Hayes et al., 2006).

Defusion is a part of all ACT interventions and a number of successful protocols emphasize defusion (see Bach \& Hayes, 2002). Top-down dismantling studies, demonstrating that defusion is an active component in successful ACT interventions, have yet to be conducted. Dismantling studies require large samples and substantial resources to conduct. As such, studies working from the bottom-up, examining whether specified techniques produce predicted changes can help fill the gap. This bottom-up approach is consistent with calls for identifying evidence-based techniques or "kernels" - specific procedures that are shown to influence a variable of interest (Embry \& Biglan, 2008; O'Donohue \& Fisher, 2008). According to the ACT model, cognitive defusion techniques should influence psychological flexibility (Hayes et al., 2006).

ACT offers a wide range of strategies for defusing from negative thoughts (see Hayes et al., 1999; Hayes \& Smith, 2005). The strategy with the most empirical support is Titchener's repetition, one of the vocalizing techniques, which involves saying a word aloud over and over again with increasing rapidity for a period of about 20-30 seconds. A common example involves asking someone to say "milk". A single utterance of "milk" is likely to result in contact with the conditioned perceptual (e.g., it's a white liquid) and sensory (e.g., its taste, texture, and temperature) properties of milk and a range of its attributes (e.g., typically comes from cows, often goes on cereal). That is, one experiences (to some extent) the presence of milk even though no milk is actually present. However, repeat "milk" over and over again at an increasing rate, and the conventional meaning and functions of the word fade and all that is left is the odd sound. The word has been temporarily deliteralized, stripped of its conventional meaning. Can this strategy be applied to negative self-thoughts? Will such a practice lead to one experiencing negative selfthoughts as merely sounds stripped of their conventional meanings and the associated aversive functions? Initial empirical studies are promising.

Vocal repetition has been applied to negative self-thoughts in several studies. Using a single-case alternating treatments design, Masuda, Hayes, Sackett, and Twohig (2004) examined the impact of vocal repetition compared to distraction and thought control conditions with eight nondistressed undergraduates. Thirty seconds of vocalizing a single personally relevant negative thought (e.g., stupid) resulted in larger immediate decreases in emotional discomfort and believability compared to the control conditions. In a follow-up parametric study, large normative collegian samples were used to demonstrate that 20-30 second repetition durations were better than shorter durations to decrease both the emotional discomfort and believability of the single personally relevant negative thought targeted (Masuda et al., 2009). Together these data suggest that, among nonclinical samples, vocalizing can immediately and at least temporarily reduce the negative aspects of taking thoughts literally.

The purpose of the present study is to test the effects of vocalizing strategies with a distressed sample. Specifically examined was the effect of a three session cognitive defusion (CD) protocol (centered around the use of vocalizing strategies) compared to a waitlist (WL) control condition applied 
to psychologically distressed collegians. This technique evaluation trial is more focused than a full-scale ACT intervention in that its emphasis is strictly on defusion. However, the intervention is more extensive that those used in the work of Masuda and colleagues allowing for application to a more clinically analogous sample, seen in the context of a treatment setting and over a time frame that allowed for targeting multiple negative self-thoughts, such that the effects on broader indices of functioning could be examined. In addition, the use of archival data from Clore and Gaynor (in press) allowed the results to be benchmarked against a comparison sample of distressed collegians following three sessions of supportive therapy.

Of particular interest were the following hypotheses: (a) CD would produce superior effects compared to a concurrent WL comparison group, (b) WL participants would show similar improvements to those initially randomized to $\mathrm{CD}$ when it was subsequently provided to them, and (c) The effect sizes for $\mathrm{CD}$ would be larger than those found in a supportive therapy comparison condition established from archival data.

\author{
Method
}

\title{
Participants
}

From November 2007 to May 2008, undergraduate students from a large midwestern public university responded to fliers and classroom announcements for free research therapy for low self-esteem and negativity. The study procedures and consent process were reviewed and approved by the Human Subjects Institutional Review Board of the university where the study took place. Of 28 potential participants assessed for eligibility, 6 were excluded. Five of those excluded failed to score one SD above the mean according to the adult nonpatient norms on the Brief Symptom Inventory (Derogatis, 1993) and one SD below the mean for a college population on the Rosenberg Self-Esteem Scale (Rosenberg, 1989; Vispoel, Boo, \& Bleiler, 2001). The sixth exclusion was due to current participation in ongoing alternative psychotherapy. Those receiving pharmacotherapy were enrolled if they had been on the same medication regime for at least 8 weeks and experienced no change in medication during the course of treatment. The 22 qualifying participants were randomly assigned (stratified by gender) to one of two conditions: $\mathrm{CD}(n=10)$ or WL $(n=12)$ and comprise the intent-to-treat sample (see Figure 1$)$. This sample size was close to our targeted enrollment size of 25. The approximate sample size for the study was based on power analyses using the syntax and approach of D'Amico, Neilands, and Zambarano (2001) for repeated measures designs. Based on estimates of change from prior work and available psychometric data the power analyses suggested that significant time*condition interactions would be found on all dependent measures with a sample size of 25 (and on 2/3 dependent measures with a sample size of 20). 


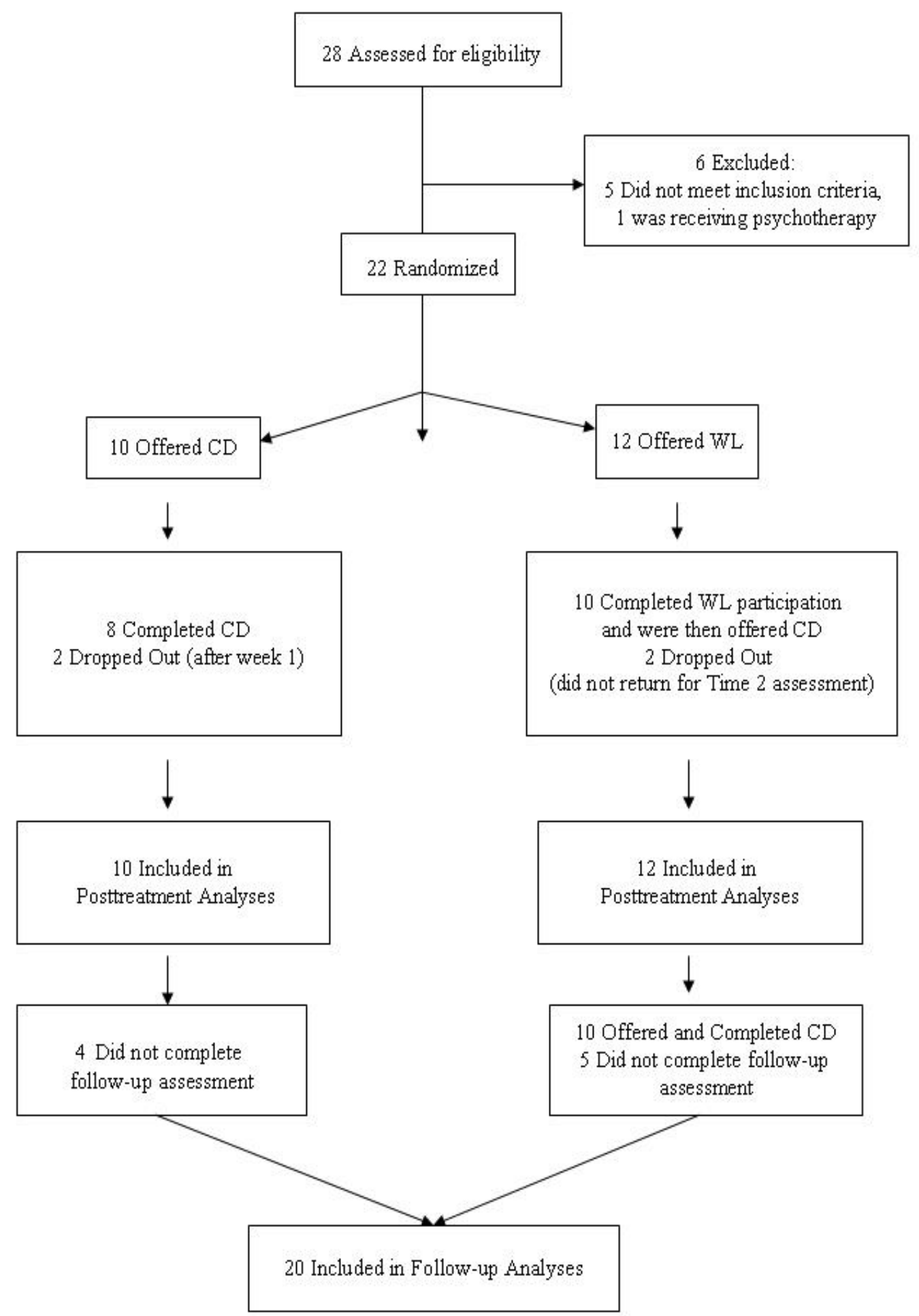

Figure 1. Participant Flow Diagram. $\mathrm{CD}=$ cognitive defusion, $\mathrm{WL}=$ waitlist condition. 
The average age of participants was $20.09(S D=2.56)$ and the majority were female $(n=16$, $73 \%)$, Euro-American $(n=19,86 \%)$, and full-time college students $(n=21,96 \%)$. Half of the sample was currently employed $(n=11)$, two participants $(9 \%)$ were currently taking psychotropic medication, and five $(23 \%)$ had a history of taking psychotropic medications. Eight participants (36\%) had a history of mental health treatment for family problems, anxiety, child abuse, or, in 4/8 cases, mood problems depressive or bipolar disorder. Table 1 presents the demographic variables for each group. There was a significant difference on only one variable, with more of those in the WL condition having a history of mental health treatment, ${ }^{2}=5.51, p=.019$. There were no significant differences on any of the other demographic variables $(p s=.43-1.00)$ nor were there significant differences between the two groups on any of the pretreatment clinical measures (see Table 2). Finally, the sample means on the BDI, BSI, and RSES were all more than 1.5 SDs from established college student norms (range 1.60-1.93 SDs) indicating that the inclusion criteria yielded a clearly distressed sample.

The supportive therapy benchmark was based on data from 27 participants $(M$ age $=21.4,70 \%$ Euro-American females) following three sessions of supportive therapy emphasizing the exploration of feelings. These 27 participants were recruited from the same midwestern university as the current sample, were enrolled based on the same inclusion criteria, were randomly assigned to supportive therapy, and completed an almost identical battery of assessment measures. The most relevant difference is that the supportive therapy participants completed the AAQ, not the AAQ-II, as the latter was not psychometrically evaluated at the time Clore and Gaynor's trial was planned and executed. See Clore and Gaynor (in press) for a detailed description of this study and its findings.

Table 1 Sample Demographic Variables

CD WL

$\underline{(n=10)} \quad \underline{(n=12)}$

$\begin{array}{lllll}\text { Variable } & n & \% & n & \%\end{array}$

Gender (Female)

Euro-American

African-American

Multiethnic

Full-time student

Employed
8

8

1

1

10

5
80

80

10

10

100

50
8

11

0

1

11

6
67

92

0

8

92

50 
History of mental health treatment

$$
\begin{array}{rrr}
\text { Treatment focus }=\text { depression } & 1 & 2 \\
=\text { bipolar disorder } & 0 & 1
\end{array}
$$

$\begin{array}{llllll}\text { Currently taking psychotropic medication } & 1 & 10 & 1 & 8\end{array}$

History of medication

$2 \quad 20 \quad 3 \quad 25$

$M \quad S D \quad M \quad S D$

$\begin{array}{lllll}\text { Age } & 19.60 & 1.35 & 20.50 & 3.26\end{array}$
Cumulative GPA
3.19
0.56
3.00
0.67

Statistically significant, $p<.05$

Table 2

Pretreatment Clinical Characteristics for CD and WL and Corresponding Data from Archival

\begin{tabular}{|c|c|c|c|c|c|c|c|c|}
\hline \multirow[b]{2}{*}{ Measure } & \multicolumn{2}{|c|}{$C D(n=10)$} & \multicolumn{2}{|c|}{$\mathrm{WL}(\mathrm{n}=12)$} & \multicolumn{2}{|c|}{ CD v. WL } & \multicolumn{2}{|c|}{ ST $(n=27)$} \\
\hline & $M$ & $S D$ & $M$ & $S D$ & $F(1,20)$ & $p$ & $M$ & $S D$ \\
\hline RSES & 22.40 & 3.09 & 22.92 & 3.06 & .15 & .699 & 22.04 & 3.01 \\
\hline BSI & 1.64 & .59 & 1.48 & .49 & .49 & .490 & 1.60 & .59 \\
\hline BDI-II & 19.80 & 5.92 & 24.17 & 5.62 & 3.14 & .092 & 22.67 & 9.41 \\
\hline AAQ-II & 39.10 & 9.07 & 39.42 & 10.52 & .01 & .941 & $\mathrm{~N}$ & \\
\hline Defusion & 11.00 & 2.54 & 11.17 & 2.21 & .03 & .871 & $\mathrm{~N}$ & \\
\hline
\end{tabular}
Supportive Therapy Condition 


$\begin{array}{lcccccccc}\text { Slippage } & 13.40 & 2.99 & 12.08 & 3.09 & 1.02 & .324 & \text { NA } \\ \text { ATQ } & 75.90 & 22.01 & 77.67 & 19.23 & .04 & .843 & 80.33 & 21.88 \\ \text { ATQ-P } & 76.60 & 14.24 & 74.83 & 12.30 & .10 & .758 & 71.11 & 21.04 \\ & & & & & & & & \\ \text { Imp Mgmt } & 5.80 & 3.16 & 6.42 & 3.18 & .21 & .654 & 6.89 & 3.62\end{array}$

Note. $\mathrm{CD}=$ Cognitive defusion; $\mathrm{WL}=$ Waitlist; $\mathrm{ST}=$ Supportive theapy; RSES = Rosenberg Self-Esteem Scale; BDI-II = Beck Depression Inventory-II; BSI = Brief Symptom Inventory, Global Severity Index; AAQ-II = Acceptance and Action Questionnaire-II; Defusion $=$ Defusion subscale, Slippage $=$ Slippage subscale, ATQ = Automatic Thoughts Questionnaire; ATQ-P = Automatic Thoughts Questionnaire-Positive, Imp Mgmt = Impression Management.

\section{Design}

A between-groups randomized controlled design was utilized wherein participants were stratified by gender and then randomly assigned to either CD or WL. The randomization sequence was determined prior to enrolling any participants by the authors using www.ResearchRandomizer.com. When a participant met inclusion criteria and consented to participate, the first author consulted a spreadsheet containing the predetermined sequence to determine group assignment. Those enrolled in CD were offered a total of three weekly therapy sessions, each approximately 1 hour in duration, to begin immediately. Those participants randomized to WL completed assessments at the same interval as those who received $\mathrm{CD}$, after which they were offered CD.

\section{Conditions}

All sessions were conducted in therapy rooms located in a research suite of a large midwestern public university department of psychology. Each therapy session was videotaped via a wall-mounted camera for the purpose of coding treatment adherence. There were no adverse events reported.

Cognitive defusion. $\mathrm{CD}$ consisted of three weekly, 1-hour, individual treatment sessions and three 30-minute assessment sessions (Time 1: pretreatment, Time 2: posttreatment, and 1-month follow-up). The treatment was guided by a detailed protocol arranged by (and available from) the authors. The protocol was heavily influenced by Hayes et al. (1999) and Hayes and Smith (2005).

The overall goal of $\mathrm{CD}$ was to enable participants to notice negative thoughts without becoming fused to, or stuck on, them. A rationale for taking this approach to negative thoughts was presented and the goal was pursued by training and practicing vocalizing strategies. Session 1 began with a didactic and experiential rationale designed to help foster a context of openness to experiencing negative thoughts wherein the vocalizing strategies could be meaningfully employed. First, low self-esteem was described as involving a tendency to treat negative self-thoughts as facts, thereby enhancing their potency. This process was illustrated experientially by inviting the participant to close his/her eyes and really "get in touch" with a personally important and highly believable negative self-thought. The present-moment impact of the thought on his/her feelings, motivation, behavioral tendencies, and general self-concept was then reviewed to illustrate the aversive functions prompted simply by having the thought. Thought control 
was then discussed as a logical but ineffective strategy for dealing with negative thoughts. The problem with simple thought control (i.e., just deciding not to have the thought anymore) was illustrated by asking the client to not think about chocolate chip cookies straight out of the oven, warm and chewy, with melting chocolate chips (as presented in Hayes et al., 1999), which they were unable to do. The role of language in contributing to fusion was also described and illustrated by asking the participant to respond to the question "Who are you?" The participant inevitably responded with his/her name allowing the therapist to point out how words are used to refer to things, but words are not the same as the things to which they refer. A person is not his/her name but because we use words to refer to things, it is easy to start treating the words as if they are the things to which they refer - the essence of fusion. There is little harm in fusing with "I am Scott" but fusing with "I am worthless" is another matter, especially because the latter illustrates how language can make subjective evaluations appear as objective descriptions. Thus, low self-esteem was presented as a byproduct of understandable aspects of our language system in which words are treated as though they are synonymous with things and evaluations are treated as literal descriptions. As such, the therapist indicated that this treatment will focus on noticing and observing negative thoughts for what they are (sounds organized into words that are given conventional meanings) and that one can have them without buying into or getting stuck on them. It was noted that this process will involve being creative with language. At this point the Milk, Milk, Milk (or, more commonly given our college population, the Beer, Beer, Beer) exercise (as outlined in Hayes et al., 1999 and described in the Introduction) was introduced. This exercise illustrated how the literal meaning of a word can be temporarily removed via repeated rapid vocalizing (20-45 seconds was the targeted duration) leaving only the funny sound and revealing the word for what it is - simply a combination of sounds. This process of vocal repetition was then conducted with a highly important and believable negative self-thought. Afterward the same negative self-thought was written on an index card along with two additional personally important and believable negative self-thoughts. Time permitting, vocal repetition was also conducted with these two negative self-thoughts. All three were then used in the Contents on Cards exercise (Hayes et al., 1999) wherein the participant practiced holding the index cards as far away from his/her body as possible, followed by simply placing the cards in his/her lap. This exercise illustrated the additional effort required when one struggles to keep troubling thoughts away versus being able to have them without buying into them. For homework, participants were asked to carry the three index cards everywhere they went throughout the week, providing a daily demonstration that they are able to possess their negative thoughts without restricting behavior or having to control them. The index cards also allowed for structured vocalizing practice to be assigned. Participants were asked to take out the index cards and practice the vocal repetition strategy with each negative self-thought three times a day. To further prompt and structure the practice, the negative thoughts were also written on a tracking form (The Choose Not To Fuse Log) where the participant was asked to record his/her use and practice of vocalizing strategies outside of session.

The second and third therapy sessions consisted of reviewing the Choose Not To Fuse Log, discussing "sticky" experiences with negative thoughts during the prior week, and having the therapist and participant practice vocalizing strategies. Practice was focused first on previously identified negative self-thoughts adding other vocalizing strategies beyond vocal repetition. These additional vocalizing strategies, from Hayes and Smith (2005) and Hayes et al. (1999), included: say it slowly, sing it, say it in a different voice, say it as the announcer of a radio station called "bad news radio", and say it as a story (a brief story about having the thought and experiencing a range of outcomes, e.g., boring, outrageous, hilarious, and terrible). If possible, participants practiced in-session using all of the vocalizing strategies at least once. After exposure to all of them, the 3-4 that they perceived as most effective were generally emphasized. Sessions two and three also involved adding new negative thoughts for targeted in-session defusion. Typically three were added at each session. These, too, were written on index cards for the participant to carry wherever he/she went and added as part of the extra-session homework practice. 
Waitlist condition. WL participants completed the Time 1 (pretreatment) assessment and then were scheduled to meet with the researcher again in approximately 3 weeks for the Time 2 assessment. The second assessment corresponded to the post treatment assessment for those in CD. Following the Time 2 assessment WL participants were offered CD, after which they completed a Time 3 assessment and a 1-month follow-up.

\section{Treatment Integrity}

The first author, a graduate student in an APA-accredited clinical psychology doctoral program, served as the therapist for all participants. The therapist, an African-American female, had didactic and clinical experience with ACT. In addition, she engaged in study-specific preparation which included detailed reading and discussing of Hayes, Strosahl, and Wilson's (1999) chapter entitled "Building Acceptance by Defusing Language" from Acceptance and Commitment Therapy: An Experiential Approach to Behavioral Therapy, and Hayes and Smith's (2005) chapters entitled "The Trouble With Thoughts" and "Having a Thought Versus Buying a Thought" from Get Out of Your Mind and Into Your Life. She also watched and discussed the Cognitive Defusion videodisc from the ACT in Action (Hayes, 2007) series. These discussions were conducted with the second author, a Ph.D. licensed psychologist who has attended several ACT trainings and has experience using ACT in clinical and research settings, and resulted in the $\mathrm{CD}$ protocol used in this study. The therapist then conducted role-play practice sessions with graduate student colleagues, feedback from which culminated in the final CD protocol. The second author provided weekly supervision throughout the study.

A measure was developed by the researchers to assess competence and adherence to the protocol. This Treatment Integrity Questionnaire was completed by the therapist after each therapy session. In addition, $32 \%$ of the session tapes were viewed by an independent doctoral student coder. Participant videotapes from the intent-to-treat sample were quasi-randomized so that each of the three sessions was equally represented across participants and no more than two sessions for any particular participant was assessed. Individual items were rated on a 6-point scale with $1=$ "not at all" and $6=$ "extensively" and combined into four subscales: cognitive defusion adherence, cognitive therapy adherence, behavior therapy competence, and overall cognitive defusion competence. The adherence subscales captured the extent to which the therapist employed techniques prescribed by the protocol (cognitive defusion adherence) and avoided using techniques not prescribed by the protocol (cognitive therapy adherence) (Waltz, Addis, Koerner, \& Jacobson, 1993), while behavior therapy competence denoted the skillfulness of the delivery of the protocol (e.g., providing the rationale in a sensible way, using relevant examples, ensuring client understanding, demonstrating engagement and attentiveness, employing Socratic questioning, establishing and following an agenda, and reviewing homework) (Waltz et al., 1993). One item provided a rating of overall cognitive defusion competence.

Agreement. Agreement between raters was calculated using an intraclass correlation coefficient, which demonstrated significant agreement $(N=393$, ICC $=.80, p<.000)$.

Adherence and competence. Ratings of 4 or above were considered representative of adherence and competence. The therapist's average rating of CD adherence was $4.68(S D=.60)$ and the coder's average was $4.29(S D=.61), F(1,35)=3.60, p=.06$, while the $\mathrm{CT}$ adherence rating by the therapist was $1.07(S D=.14)$ and the coder's average was $1.17(S D=.24), F(1,35)=2.03, p=.16$. With respect to general behavior therapy competence, the therapist's average rating was $4.24(S D=.68)$ while the coder's rating was $4.22(S D=.58), F(1,35)=.06, p=.94$. CD competence as rated by the therapist $(M=4.83, S D$ $=.38)$ and independent coder $(M=5.83, S D=.38)$ both indicated competence but were significantly different, $F(1,35)=61.2, p=.00$, with the coder reporting greater competence. Overall these data suggest that $\mathrm{CD}$ was delivered competently with strong adherence to the protocol. 


\section{Measures}

Measures included a demographic and educational status questionnaire, an impression management scale (Paulhus, 1991), seven commonly used clinical inventories, and a self-thought fluency assessment (STFA) developed by the authors. While a participant was enrolled in the study, the therapist was kept blind to all assessment measures except the initial Rosenberg Self-Esteem Scale (RSES) and the Brief Symptom Inventory (BSI), which were required for determining eligibility, and the self-thought fluency assessment (STFA), I don't believe this should be capitalized especially insofar as there is no citation suggesting that it has been published which was used in guiding the selection of negative selfthoughts for use in the first session. The STFA was developed and used previously by the authors. It involves two separate 3-minute periods in which the participant is given 2 minutes to collect his/her selfthoughts, and then 1 minute to write as many self-positives or self-negatives as she/he can. After the positive and negative thoughts are generated, each is rated for both personal importance and believability.

Outcome measures. The RSES, BSI, and Beck Depression Inventory-II (BDI) are commonly used indices of psychological symptoms/states and were considered the primary measures of outcome.

The 10-item RSES (Rosenberg, 1989) asks participants to rate their agreement (from $0-4$ ) with statements describing general feelings about themselves. Higher scores indicate a more positive selfevaluation, with a mean of $32.60(\mathrm{SD}=5.25)$ established in a large nonpatient college sample (Vispoel et al., 2001). Alpha coefficients have ranged from .72 to .88 and test-retest coefficients of .82 and .67 have been reported over 1-week and 7-month time intervals, respectively (Wylie, 1989).

The BSI (Derogatis, 1993) is a 53-item questionnaire assessing psychological symptoms on a 5-point Likert scale from 0 (not at all) to 4 (extremely), with higher scores indicating more distress. The Global Severity Index (GSI) has been identified as the most sensitive single indicator of psychological distress (Derogatis, 1993). Normative GSI means with college students of $0.84(\mathrm{SD}=0.55)$ for males and 0.71 $(\mathrm{SD}=0.42)$ for females were reported by Cochran and Hale (1985). Alpha coefficients for the BSI's dimensions have ranged from .71 to .85 in an outpatient sample and a GSI test-retest reliability of .90 over a 2-week time interval has been reported for non-patients on the GSI (Derogatis, 1993).

The BDI-II (Beck, Steer, \& Brown, 1996), a 21 -item self-report scale, assesses the severity of depressive symptoms. Items consist of four self-evaluative statements scored 0 to 3 , with increasing scores indicating greater levels of depression. Normative means from two large collegiate samples were 8.36 (SD = 7.16; Whisman, Perez, \& Ramel, 2000) and 9.11 (SD = 7.57, Dozois, Dobson, \& Ahnberg, 1998). High levels of internal consistency (.89 to .93) and high short-term test-retest reliability have also been reported (Beck et al., 1996; Sprinkle et al., 2002; Whisman et al., 2000).

Process measures. Psychological flexibility and negative thinking are common targets of change in cognitive behavior therapy and theoretically are typically considered mechanisms of action by which treatment works (Kanter, Baruch, \& Gaynor, 2006). As such, the Acceptance and Action Questionnaires and Automatic Thoughts Questionnaires were considered process measures.

The Acceptance and Action Questionnaire-II (AAQ-II; Bond et al., submitted) is a 10-item scale measuring the ability to take action despite uncomfortable thoughts/feelings. It is a second generation version of the original AAQ (see Hayes et al., 2004) and is considered a primary index of psychological flexibility as presented in the ACT model. Both the AAQ-II and AAQ (9 item version) are scored on a 1-7 scale. On the AAQ-II higher total scores represent greater psychological flexibility, with a normative mean score of $50.72(S D=9.19)$ for college students. Across seven samples, a high level of internal consistency has been reported with coefficient alphas ranging from .76 to .87. On the AAQ, lower total scores, representing less experiential avoidance, are more favorable with a mean for non-clinical populations of $33.4(\mathrm{SD}=7.2)$. 
The 30-item Automatic Thoughts Questionnaire (ATQ; Hollon \& Kendall, 1980) measures the frequency of negative self-statements. Each item was scored on a 5-point scale (ranging from 1-not at all to 5-all the time) with higher scores indicative of more negativity. Nonpatients score between 40 and 60 $(\mathrm{M}=52.91, \mathrm{SD}=18.18$ ), while depressed patients tend to score over 90 (Dozois, Covin, \& Brinker, 2003). The ATQ has been reported to have high reliability $(\alpha=.96)$ and is considered a useful tool in assessing change in cognitions related to psychotherapeutic intervention (Hollon \& Kendall, 1980).

The 30-item Automatic Thoughts Questionnaire-Positive (ATQ-P; Ingram \& Wisnicki, 1988) measures the frequency of positive self-statements (e.g., "I'm fun to be with.") and is scored from 1 (not at all) to 5 (all the time), with higher scores indicating more positivity. As reported by Ingram and Wisnicki (1988), the mean score for nondepressed individuals is $107(\mathrm{SD}=19), 96(\mathrm{SD}=19)$ for mildly depressed, and $83(\mathrm{SD}=16)$ for depressed individuals. However, the normative mean averaged across samples, as reported by Dozois et al. $(2003)$ was $98.61(\mathrm{SD}=13.02)$. Excellent internal reliability $(\alpha=$ .95) has also been reported.

Repeated measure. The 10-item Mini Cognitive Defusion and Slippage Scale was constructed from the Freiburg Mindfulness Inventory (Buchheld, Grossman, \& Walach, 2002) and Cognitive Slippage Scale (Miers \& Raulin, 1985). The latter assesses confused thinking often seen in schizophrenia spectrum disorders. Participants completed the measure at Time 1, 2, 3, and prior to each therapy session when receiving $C D$. The 10 -items on rated on a 1 (rarely true) to 4 (usually true) Likert scale and were arranged in random sequence. Administering the scale in this way allowed for an examination of the specificity of change across repeated administrations. That is, would participants report similar changes on both subscales (as may be the case simply due to repeated administrations)? Or, as predicted by the ACT model, would there be differential increases on the Defusion subscale (which tapped defused awareness of thoughts) but no group differences on the Slippage subscale. For the Defusion subscale, the five items with the highest item-to-total correlations from the Freiburg Mindfulness Inventory (items 2, 11, 18, 22, and 25) were identified (Buchheld, Grossman, \& Walach, 2002). These items were then modified to focus solely on thoughts and to be in the past tense, allowing the instructions to sample experiences over the past week. The resulting items were: I was able to observe my thoughts without having to react to them, I was able to accept unpleasant thoughts, I could look at my thoughts without identifying with them, I noticed my thoughts without becoming lost in them, and I recognized that I am not identical to my thoughts. Based on the Time 1 data (the time point at which there was the most data available), the items did not demonstrate strong internal consistency $(N=22,=.48)$. However, such a result is not unexpected given that the scale had a small number of items, was given to a very small sample for psychometric purposes, and attempted to assess a fairly broad construct, all of which are factors that influence Cronbach's alpha. Despite the modest alpha, the scale appeared to function as intended, correlating significantly with the AAQ $(r=-.57, p=.02)$ and AAQ-II $(r=.61, p=.003)$, but not the BSI $(r=-.20, p=.39)$, BDI-II $(r=-.39 ., p=.07)$, RSES $(r=.18, p=.42)$, ATQ $(r=-.37, p=.09)$, ATQ-P $(r$ $=.07, p=.77)$, or Slippage $(r=.32, p=.14)$. For the Slippage subscale, five items related to misperceptions, haziness, and rapidity of thought were selected (items 10, 13, 25, 31, and 32) and were reworded in the past tense.

\section{Data Analysis}

Pretreatment differences between the two conditions were analyzed using Pearson's chi-square tests and one-way ANOVAs. The between-groups outcome analyses were conducted using a mixed models framework (SPSS Linear Mixed Models procedure) with the intent-to-treat sample. This framework was selected because it allowed inclusion of cases with incomplete data and does not require the assumption of constant variance, features which recommend its use with repeated measures designs (Norušis, 2008). A model with correlated terms was specified with participants treated as a cluster of independent cases (subject variable) and time as the repeated variable. Treatment, time, and the 
treatment*time interactions were entered as fixed factors and participants were included in the model as a subject grouping. All analyses were run using unstructured, compound symmetry, and first-order autoregressive covariance structures to determine which produced the best fit, determined by comparing 2 log-likelihood (-2LL), Akaike information criterion (AIC), and Bayesian information criterion (BIC) statistics. The data from the covariance structure with the best goodness-of-fit were selected for presentation (Norušis, 2008). All outcome and process measures were analyzed individually resulting in a large number of analyses increasing the risk of a Type I error. However, given the consistent pattern of findings (reducing the likelihood of generating misleading conclusions based on an isolated spurious finding) and the relatively small sample size (increasing the risk of a Type II error), a Bonferroni correction to the conventional alpha level of .05 was not employed. Between-group effect sizes were also calculated, using Hedges $g$ statistic (which is recommended for small sample sizes) based on the full sample standard deviation.

All 10 of the participants who completed participation in the WL condition, went on to receive and complete $\mathrm{CD}$. These data provide an opportunity to see if the effects of CD were able to be replicated. To examine whether CD yielded more change than being in WL, a series of paired samples t-tests were conducted comparing change from Time 1 to Time 2 (the WL interval) and Time 2 to Time 3 (during which they received $\mathrm{CD}$ ) using the intent-to-treat sample with last data point carried forward for those who did not return to receive $\mathrm{CD}$.

Follow-up assessments were completed 1-month after completion of CD. Of the 20 participants offered CD (either by initial randomization or after completion of WL participation), 18 completed CD and 11 participated in the 1-month follow-up assessment. The follow-up analyses used the combined intent-to-treat with CD sample $(n=20)$ and the same basic mixed models framework described above, but with time specifying three data points (i.e., preCD, postCD, and 1-month following $\mathrm{CD}$ ) and with no between-groups comparisons. The linear mixed models procedure was used to establish the estimated means at each time point from which pretreatment to posttreatment and posttreatment to follow-up within group effect sizes (using Hedges g) were calculated. The pre-treatment to post-treatment within group effect sizes for the combined CD sample were then benchmarked against the within group effect sizes from the supportive therapy group in Clore and Gaynor (2010).

\section{Results}

\section{Effect of CD versus WL on outcome measures}

As shown in Table 3 , a statistically significant time*condition interaction was found on the BSI $(F=9.32, p=.007, g=.70)$ and the RSES $(F=14.94, p=.001, g=1.45)$, suggesting superior outcome for those receiving CD and a large effect size. The BDI-II was trending toward significance $(F=3.14, p=$ .092). Because of the medium effect size between $C D$ and WL at pretreatment, change scores were also analyzed. Time 2 BDI scores were regressed on Time 1 scores to yield residualized change scores. A oneway ANOVA comparing the $\mathrm{CD}$ and WL residualized change scores was statistically significant $(F=$ $6.40, p=.02, g=1.04$ ) indicating greater change during the Timel to Time 2 interval in CD compared to WL. 
Table 3 Intent-to-treat posttreatment outcome analyses

\begin{tabular}{cccccc}
\hline Measure & $\mathrm{CD}(\mathrm{n}=10)$ & WL $(\mathrm{n}=12)$ & ES & \multicolumn{2}{c}{ Time*Treatment } \\
& $E M(S D)$ & $E M(S D)$ & $g$ & $F$ & $p$
\end{tabular}

BDI

Time $1 \quad 19.80(6.54) \quad 24.20(6.54) \quad .64$

$\begin{array}{lllll}\text { Time } 2 & 11.60(7.26) & 22.40(7.12) & 1.43 & 3.14\end{array}$

$\begin{array}{llllll}(\text { Res } \Delta & -0.51(.85) & 0.43(.89) & 1.04 & 6.40 & .020)\end{array}$

Time $3 \quad 14.84(9.21)$

BSI

Time $1 \quad 1.64(0.58) \quad 1.48(0.57) \quad-.27$

$\begin{array}{llllll}\text { Time } 2 & 0.90(0.61) & 1.34(0.60) & .70 & 9.32 & .007\end{array}$

Time $3 \quad 0.82(0.53)$

RSES

Time $1 \quad 22.40(3.55) \quad 22.90(3.55) \quad-.14$

$\begin{array}{lllll}\text { Time } 2 & 28.3(3.86) & 22.50(3.80) & 1.45 & 14.94\end{array}$

Time $3 \quad 25.83(4.51)$

Note. Time 1 and 2 data are estimated means from the linear mixed models output, Time 3 data are means from the paired $t$ tests using the last data point carried forward. Res $\Delta=$ Analysis of residualized change scores using a one-way ANOVA, conducted because of the medium-sized pretreatment difference between $\mathrm{CD}$ and WL on the BDI. $\mathrm{CD}=$ Cognitive defusion; WL = Waitlist; $\mathrm{ES}=$ Effect size, Hedges $g$ with the sign adjusted so positive scores indicate better functioning in $\mathrm{CD}$, BDI $=$ Beck Depression Inventory; BSI = Brief Symptom Inventory, Global Severity Index; RSES = Rosenberg Self-Esteem Scale.

Effect of $C D$ versus WL on process variables

Both ACT-relevant process measures showed statistically significant time*condition interactions and large effect sizes favoring CD, AAQ-II: $F=6.93, p=.018, g=.81$, Defusion subscale: $F=6.84, p=$ $.013, g=.92$. The Defusion subscale data which served as a weekly repeated measure for the CD condition are particularly interesting in showing a gradual session-by-session improvement in defused awareness of thoughts (see Figure 2). There was no corresponding significant time*condition interaction on the Slippage subscale, $F=0.001, p=.98$. Instead there was a significant time effect, $F=4.47, p=$ .011 , suggesting equal movement on this measure across the entire sample. The measures of frequency of 
negative and positive self-thoughts also differed significantly in favor of CD, ATQ: $F=7.14, p=.016, g$ $=1.01$, ATQ-P: $F=11.12, p=.004, g=1.07$.
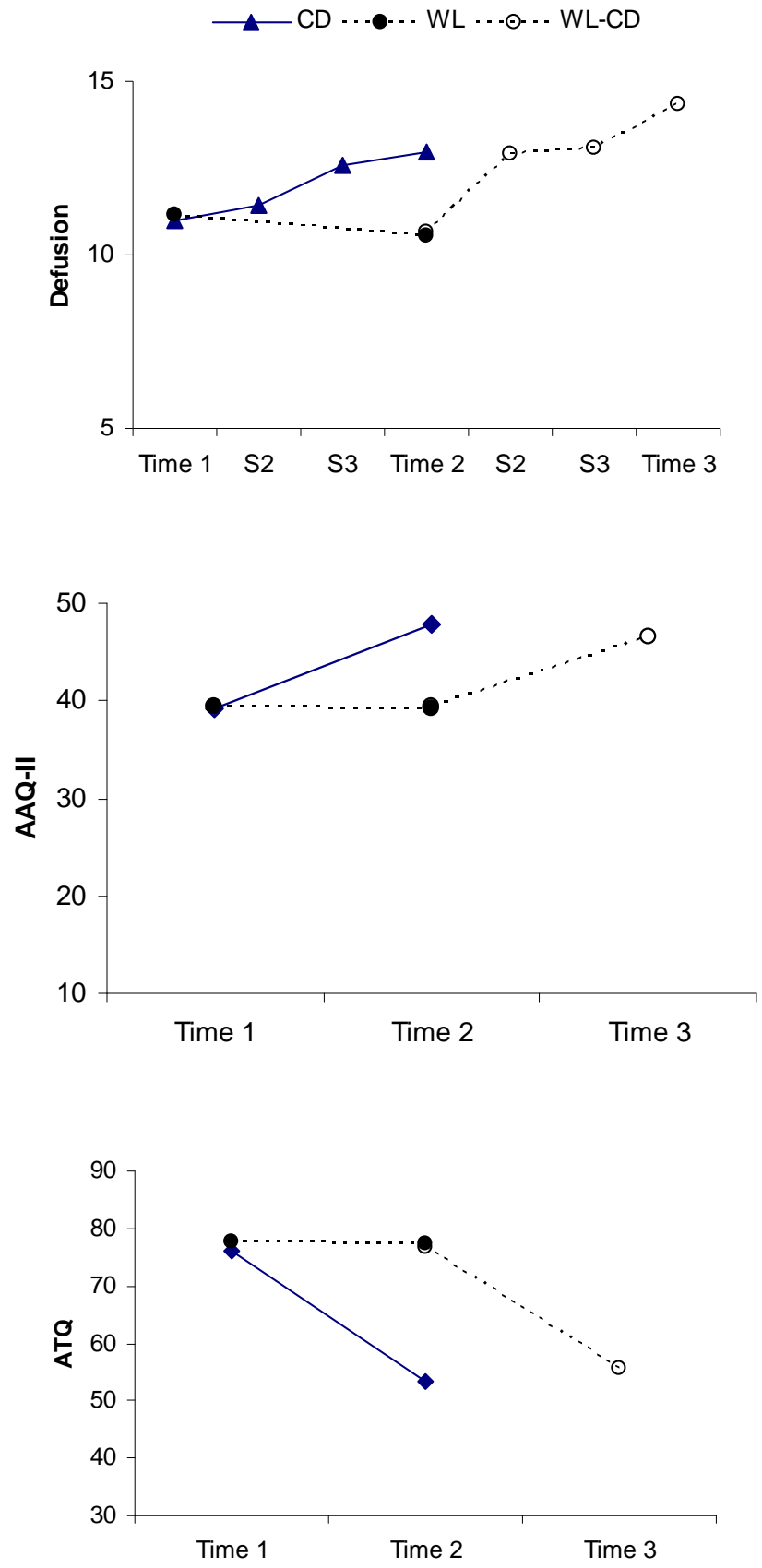


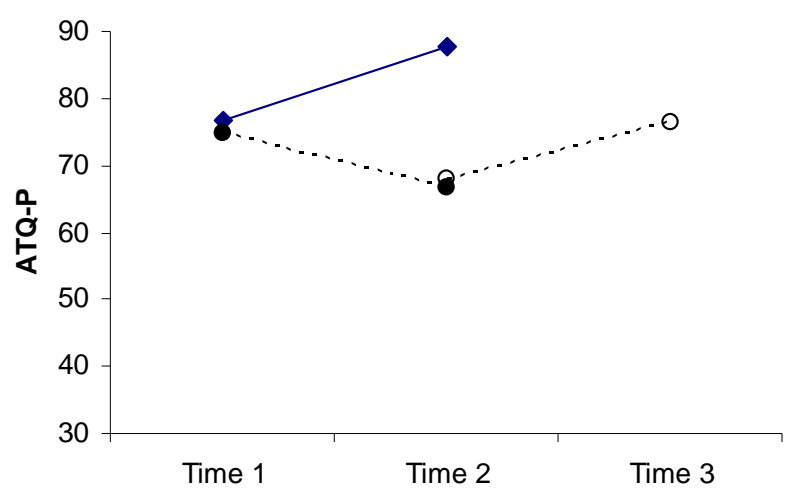

Figure 2. Time course data on process measures. $\mathrm{CD}=$ cognitive defusion, $\mathrm{WL}=$ waitlist condition, WL-CD = data from former WL participants when receiving $\mathrm{CD}, \mathrm{S} 2=$ data collected prior to session 2, S3 = data collected prior to session 3 .

\section{Replication of CD's Effects with WL Participants}

The results comparing Time 1 to Time 2 showed no significant improvements on any of the outcome or process measures, RSES: $t(1,11)=.54, p=.60$, BSI: $t(1,11)=1.35, p=.20$, BDI-II: $t(1,11)=$ $.75, p=.47$, AAQ-II: $t(1,11)=.04, p=.97$, Defusion subscale: $t(1,11)=1.07, p=.31$, Slippage subscale: $t(1,11)=-2.10, p=.059$, and ATQ: $t(1,11)=.20, p=.85$. The Time 1 to Time 2 ATQ-P data for the WL participants revealed a significant decrease in positive thoughts, ATQ-P, $t(1,11)=3.16, p=.009$. Thus, WL participants did not improve during their waiting period (See Figure 2).

From Time 2 to Time 3 the ATQ-P, $t(1,11)=-1.39, p=.19$, and the Slippage subscale, $t(1,11)=-$ $1.39, p=.059$, failed to show statistically significant change. However, comparisons of Time 2 to Time 3 functioning revealed significant improvements on the RSES: $t(1,11)=-4.16, p=.002$, BSI: $t(1,11)=$ $4.40, p=.001$, BDI-II: $t(1,11)=3.24, p=.008$, AAQ-II: $t(1,11)=-3.79, p=.003$, Defusion subscale: $t(1,11)=-4.16, p=.002$, and ATQ: $t(1,11)=3.26, p=.008$. Thus, while there were no significant changes during a 3 week waiting period, once $\mathrm{CD}$ was received significant changes on outcome and process variables comparable to those found in the original CD group were observed (see Figure 2).

\section{Maintenance of CD's Effects at 1-month Follow-up}

As shown in Table 4, within-group effect sizes based on the estimated means (for the pooled CD sample) on measures taken immediately prior to $\mathrm{CD}$, after $\mathrm{CD}$, and at 1 month follow-up from $\mathrm{CD}$, suggest large effects during the treatment period with continued small-to-moderate improvements noted at follow-up.

Table 4 Estimated means and effect sizes for the pooled $(N=20)$ intent-to-treat with CD sample

Measure

Pre CD Post CD 1-mo FU Pre-Post ES Post-FU ES

$E M(S D) \quad E M(S D) \quad M(S D) \quad g \quad g$




$\begin{array}{lrrrrr}\text { BDI } & 21.10(7.18) & 12.25(7.47) & 8.67(9.56) & 1.18 & .41 \\ \text { BSI } & 1.52(0.56) & 0.84(0.57) & 0.58(0.67) & 1.19 & .41 \\ \text { RSES } & 22.45(4.02) & 27.28(4.14) & 27.96(4.92) & 1.16 & .15 \\ \text { AAQ-II } & 39.00(9.21) & 47.69(9.44) & 48.69(10.87) & .91 & .10 \\ \text { AAQ } & 41.48(6.48) & 36.69(6.66) & 34.48(7.60) & .73 & .30 \\ \text { ATQ } & 77.25(19.68) & 53.32(20.35) & 48.41(24.60) & 1.17 & .21 \\ \text { ATQ-P } & 71.90(18.02) & 81.35(18.87) & 90.09(23.61) & .50 & .40\end{array}$

Note. $\mathrm{CD}=$ Cognitive defusion; $\mathrm{ES}=$ Effect size, Hedges $\mathrm{g}$ with the sign adjusted so that positive scores always represent change in therapeutic direction; BDI = Beck Depression Inventory; BSI = Brief Symptom Inventory, Global Severity Index; RSES = Rosenberg Self-Esteem Scale; AAQ-II = Acceptance and Action Questionnaire, second version; AAQ = Acceptance and Action Questionnaire; ATQ = Automatic Thoughts Questionnaire-Negative; ATQ-P = Automatic Thoughts QuestionnairePositive.

\section{Benchmarked Against a Comparable Amount of Supportive Therapy}

As presented in Figure 3, within group effect sizes for three sessions of CD were larger than those established during three sessions of supportive therapy on all of the outcome and process measures. While preliminary, these data suggest that the effects of $\mathrm{CD}$ are beyond those attributable to the provision of a comparable amount of therapeutic support.

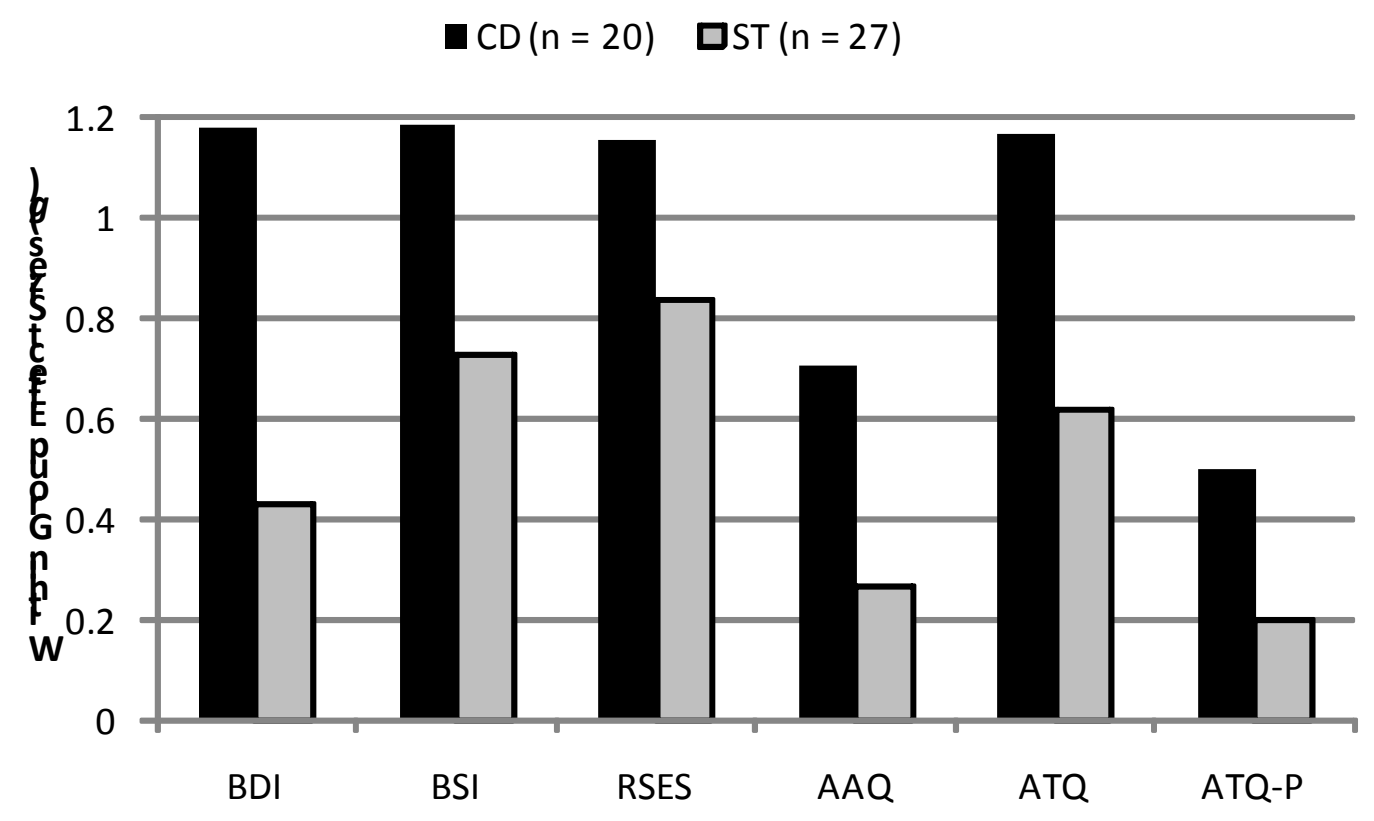

Figure 3. Within group effect sizes (Hedges g) after three sessions for the pooled CD group and the supportive therapy condition from Clore and Gaynor (2010). 


\section{Discussion}

The available data support the following conclusions: Three sessions of CD produced clearly superior change on outcome and process measures compared to no treatment and appeared better than provision of an equal amount of contact with a supportive therapist. Moreover, the process variables targeted by CD changed as expected over the course of treatment. CD participants reported greater psychological flexibility at the conclusion of treatment and the session-by-session measure of defused awareness of thoughts suggested gradual acquisition of this skill. These data are consistent with the ACT model (Hayes et al., 2006). Furthermore, the present data fit nicely in an open space between existing bottom-up and top-down ACT studies. Masuda and colleagues $(2004,2009)$ showed vocal repetition of a single negative thought led to immediate decreases in the discomfort and believability of that thought among a nondistressed sample; however, the focus and design of those studies did not allow for broader effects to be analyzed. Other studies demonstrate the efficacy of multicomponent ACT protocols (see Hayes et al., 2006) for a range of problems; however, those studies have not allowed for the effects of specific components to be analyzed. Because of the nature of the existing literature, the active role of acceptance practices in the change process has been questioned and it has been suggested that "component and meditational analyses would help address the utility of cognitive defusion and acceptance" (Arch \& Craske, 2008, p. 267). The present study contributes directly in this area helping fillin the existing literature and furthering the database suggesting cognitive defusion is a likely active ingredient in ACT.

CD not only influenced psychological flexibility and mindful awareness of thoughts, but also clearly produced decreases in negative thinking. This is not an uncommon finding in existing ACT studies. Two small-scale depression trials comparing 12 sessions of an early iteration of ACT to partial and full CT packages found both ACT and CT produced significant and comparable decreases in the frequency of negative thoughts on the ATQ (Zettle \& Hayes, 1986; Zettle \& Rains, 1989). Thus, despite the fact that there is no explicit emphasis on decreasing the frequency of negative thoughts in ACT (or $\mathrm{CD})$, that such a change occurs appears to be a relatively common effect.

Because cognitive defusion does not explicitly target the content or frequency of negative thoughts for change, how is it that defusion might produce a decrease in the frequency of negative thoughts? As discussed in the Introduction, negative self-thoughts do not appear in isolation, but rather often co-occur, occur in conjunction with other private and public aversives, and occur in a broader social/verbal context where their presence is considered indicative of abnormality. Consider the example of a participant who had the thought, "I'm not good enough". This negative thought typically had companions, such as "I'm not funny, I'm not outgoing, I'm not normal, I'm socially awkward", which would often recur over multiple iterations. However, when "I'm not good enough" was sung (repeatedly to the tune of "Just the Two of Us" by Grover Washington, Jr. and Bill Withers) the typical chain of ruminative negative thoughts would be preempted. If the typical relational network of negative selfthoughts was regularly preempted in this fashion via vocalizing strategies, this would indeed produce a decrease in the overall frequency of negative thoughts. Similarly, "I'm not good enough" often had other associated aversive functions, such as generating further negative affect/emotional distress and worry about self-esteem. The negative affect and worry about self-esteem itself became problematic because having these experiences represented "evidence" of abnormality (something for one to worry about) and further supported a literal interpretation of the original negative thought. Again, if singing "I'm not good enough" disrupted or weakened these associated aversive functions (e.g., such that there was limited or no emotional distress, and subsequent worry about self-esteem and thoughts of being disordered were not generated), decreases in both negative thinking and internalizing symptoms would be expected.

There are a number of limitations to the present study that could be addressed in future research. For one, there was no index of overt behavior change outside the session and all of the assessments were 
self-report measures completed by the participant. The assessment battery did not include clinical interviews, either to establish diagnostic status or to have an independent evaluator further document the symptomatic changes reported. With respect to the lack of a blind assessor, procedures were put in place, about which participants were made aware, so that therapists were blind to all measures except the pretreatment RSES, BSI, and STFA. As for the lack of diagnostic determination, the goal of the present study was not to offer CD as a stand-alone treatment for any particular clinical condition. That said, the dysfunctional nature of the current sample's pretreatment scores according to available norms for the outcome and process measures, as well as the use of CD techniques in other ACT protocols with diagnosed clinical samples, provide some optimism that CD techniques are clinically applicable.

Another limitation is that the measures used were all collected concurrently at Time 1 and Time 2 precluding temporally sensitive analyses of whether the process variables served as mediators of treatment outcome. Findings of statistical mediation are strongest when the process variable is measured before the outcome variable and are especially strong if the measured change in the process variable occurs before a significant amount of change on the outcome variable (Kazdin \& Nock, 2003; Kraemer, Wilson, Fairburn, \& Agras, 2002; Gaynor \& Harris, 2008). Such analyses were not possible with this dataset given the data collection procedures and the brevity of the intervention ${ }^{1}$.

Attrition during acute treatment was generally minimal with only $2 / 20$ who started CD discontinuing prior to receiving all three sessions. However, the same is not true of the follow-up data where 9/20 failed to return to complete the 1-month assessment. As such, these follow-up data must be viewed more tentatively as the functioning of the subset who elected to return may not be representative of all of those treated.

There are a number of directions for future research. Given the small sample, replication with a larger sample would be reasonable. In addition, it would be useful to evaluate CD compared to supportive therapy in a randomized comparative efficacy trial to more conclusively determine the incremental effect of $C D$ versus simply receiving therapy sessions. Other $C D$ techniques could also be examined. The $C D$ protocol used herein emphasized vocalizing strategies, but there are myriad other defusion techniques that could be studied (see Healy et al., 2008 for an example). CD sessions could also be compared directly to alternative approaches to dealing with negative thoughts, such as cognitive restructuring or increasing positive self-statements (Clore \& Gaynor, 2006) to more clearly elucidate unique effects. Another direction would be to build on the present results using an assembly model; that is, by systematically adding components. An obvious possibility would be activity scheduling (a component of behavioral activation and cognitive therapy), which could be added before or after CD in a series of studies to explore incremental efficacy and sequence effects. Adding activity scheduling is especially sensible from an ACT perspective where mindfulness strategies are ultimately linked to behavior change strategies (Hayes et al., 1999).

In conclusion, the present small-scale technique evaluation trial demonstrated that compared to a WL control, three sessions of CD (specifically training in using vocalizing strategies) with a goal of altering one's perspective on thoughts, but not the thoughts per se, increased psychological flexibility, decreased negative thinking, and reduced psychological distress, depressive symptoms, and low selfesteem. The positive results for CD were replicated when the WL group was treated. These data suggest that CD has promise as a potential evidence-based technique (Embry \& Biglan, 2008; O'Donohue \& Fisher, 2008) and support the view that defusion can be an active ingredient in ACT. Clearly much more work remains to be done as the field attempts to elucidate not only the most effective interventions, but also the processes of change engaged by these interventions (Rosen \& Davison, 2003). 


\section{References}

Arch, J. J., \& Craske, M. G. (2008). Acceptance and commitment therapy and cognitive behavioral therapy for anxiety disorders: Different treatments, similar mechanisms? Clinical Psychology: Science \& Practice, 5, 263-279.

Beck, A.T., Steer, R., \& Brown, G.K. (1996). Beck Depression Inventory - Second edition manual. San Antonio, TX: Psychological Corporation

Bond, F.W., Hayes, S.C., Baer, R.A., Carpenter, K.M., Orcutt, H.K., Waltz, T., \& Zettle, R.D. (Submitted). Preliminary psychometric properties of the Acceptance and Action Questionnaire - II: A revised measure of psychological flexibility and acceptance.

Buchheld, N., Grossman, P., \& Walach, H. (2002). Measuring mindfulness in insight meditation (vipassana) and meditation-based psychotherapy: The development of the Freiburg Mindfulness Inventory (FMI). Journal for Meditation and Meditation Research, 1, 11-34.

Clore, J. \& Gaynor, S. T. (2006). Self-statement modification techniques for distressed college students with low self-esteem and depressive symptoms. International Journal of Behavioral Consultation and Therapy, 2, 314-330.

Clore, J. \& Gaynor, S.T. (in press). Cognitive modification versus therapeutic support for internalizing distress and positive thinking: A randomized technique evaluation trial. Cognitive Therapy and Research.

Cochran, C. D. \& Hale, W. D. (1985). College student norms on the Brief Symptom Inventory. Journal of Clinical Psychology, 41, 777-779.

D’Amico, E.J., Neilands, T.B., \& Zambarano, R. (2001). Power analysis for multivariate and repeated measures designs: A flexible approach using SPSS MANOVA procedure. Behavior Research Methods, Instruments, and Computers, 33(4), 479-484.

Derogatis, L. R. (1993). Brief Symptom Inventory, administration, scoring, and procedures manual Fourth edition. San Antonio, TX: NCS Pearson, Inc.

Dozois, D. J. A., Covin, R., \& Brinker, J. K. (2003). Normative data on cognitive measures of depression. Journal of Consulting \& Clinical Psychology, 71, 71-80.

Dozois, D. J. A., Dobson, K. S., \& Ahnberg, J. L. (1998). A psychometric evaluation of the Beck Depression Inventory-II. Psychological Assessment, 10, 83-89.

Embry, D.D. \& Biglan, A. (2008). Evidence-based kernels: Fundamental units of behavioral influence. Clinical Child and Family Psychology Review, 11, 75-113.

Gaynor, S. T. \& Harris, A. (2008). Single-participant assessment of treatment mediators: Strategy description and examples from a behavioral activation intervention for depressed adolescents. Behavior Modification, 32, 372-402.

Hayes, S. C. (2004). Acceptance and Commitment Therapy, Relational Frame Theory, and the third wave of behavior therapy. Behavior Therapy, 35, 639-665. 
Hayes, S. C. (Ed.). (2007). ACT in action DVD series. Oakland, CA: New Harbinger.

Hayes, S.C., Follette, V.M., \& Linehan, M. (Eds.) (2004). Mindfulness and acceptance: Expanding the cognitive behavioral tradition. New York: Guilford.

Hayes, S. C., Luoma, J., Bond, F., Masuda, A., \& Lillis, J. (2006). Acceptance and Commitment Therapy: Model, processes, and outcomes. Behaviour Research and Therapy, 44, 125.

Hayes, S. C. \& Pankey, J. (2003). Acceptance. In: W. O’Donohue, J. E. Fisher, S. C. Hayes (Eds.) Cognitive behavior therapy: Applying empirically supported techniques in your practice (pp. 4-9). Hoboken, NJ: Wiley.

Hayes, S. C. \& Smith, S. (2005). Get out of your mind and into your life. Oakland, CA: New Harbinger.

Hayes, S. C., Strosahl, K. D., \& Wilson, K. G. (1999). Acceptance and commitment therapy: An experiential approach to behavior change. New York: Guilford Press.

Hayes, S. C., Strosahl, K. D., Wilson, K. G., Bissett, R. T., Pistorello, J., Toarmino, D., et al. (2004). Measuring experiential avoidance: A preliminary test of a working model. The Psychological Record, 54, 553-578.

Healy, H.A., Barnes-Holmes, Y., Barnes-Holmes, D., Keogh, C., Luciano, C. \& Wilson, K. (2008). An experimental test of a cognitive defusion exercise: Coping with negative and positive selfstatements. The Psychological Record, 58, 623-640.

Hollon, S. D., \& Kendall, P. C. (1980). Cognitive self-statements in depression: Development of an automatic thoughts questionnaire. Cognitive Therapy and Research, 4, 383-395.

Ingram, R. E. \& Wisnicki, K. S. (1988). Assessment of Positive Automatic Cognition. Journal of Consulting and Clinical Psychology, 56, 898-902.

Kanter, J.W., Baruch, D.E., \& Gaynor, S.T. (2006). Acceptance and commitment therapy and behavioral activation for the treatment of depression: Description and comparison. The Behavior Analyst, 29(2), 161-185.

Kazdin, A.E. \& Nock, M.K. (2003). Delineating mechanisms of change in child and adolescent therapy: Methodological issues and research recommendations. Journal of Child Psychology and Psychiatry, 44(8), 1116-1129.

Kraemer, H.C., Wilson, G.T., Fairburn, C.G., \& Agras, W.S. (2002). Mediators and moderators of treatment effects in randomized clinical trials, Archives of General Psychiatry 59, 877-883.

Masuda, A., Hayes, S. C., Sackett, C. F., \& Twohig, M. P. (2004). Cognitive defusion and self-relevant negative thoughts: Examining the impact of a ninety year old technique. Behaviour Research and Therapy, 42, 477-485.

Masuda, A., Hayes, S. C., Twohig, M. P., Drossel C., Lillis, J., \& Washio, Y. (2009). A parametric study of cognitive defusion and the believability and discomfort of negative selfrelevant thoughts, Behavior Modification, 33(2), 250-262. 
Miers, T.C. \& Raulin, M.L. (1985). Cognitive Slippage Scale. In J. Fischer \& K.Corcoran (Eds.). Measures for clinical practice and research: A sourcebook. Fourth edition, Volume 2: Adults (pp.138-140). New York, NY: The Free Press.

Norušis, M. (2008). SPSS 16.00 Guide to Data Analysis, $2^{\text {nd }}$ Ed. Upper Saddle River, NJ: Prentice Hall.

O'Donohue, W.T. \& Fisher, J.E. (Eds.). (2008). General principles and empirically supported techniques of cognitive behavior therapy. Hoboken, NJ: John Wiley \& Sons.

Paulhus, D. L. (1991). Measurement and control of response bias. In J. P. Robinson, P. R. Shaver, \& L. S. Wrightsman (Eds.), Measures of personality and social psychological attitudes. San Diego: Academic Press.

Rosen, G.M. \& Davison, G.C. (2003). Psychology should list empirically supported principles of changes (ESPs) and not credential trademarked therapies or other treatment packages. Behavior Modification, 27, 300-312.

Rosenberg, M. (1989). Society and the adolescent self-image. Revised edition. Middletown, CT: Wesleyan University Press.

Sprinkle, S.D., Lurie, D; Insko, S.L., Atkinson, G., Jones, G.L., Logan, A.R., \& Bissada, N.N. (2002). Criterion validity, severity cut scores, and test-retest reliability of the Beck Depression InventoryII in a university counseling center sample. Journal of Counseling Psychology, 49(3), 381-385.

Vispoel, W. P., Boo, J., \& Bleiler, T. (2001) Computerized and paper-and-pencil versions of the Rosenberg Self-Esteem Scale: A comparison of psychometric features and respondent preferences. Educational and Psychological Measurement, 61, 461-475.

Waltz, J., Addis, M., Koerner, K., \& Jacobson, N.S. (1993). Testing the integrity of a psychotherapy protocol: Assessment of adherence and competence. Journal of Consulting and Clinical Psychology, 61, 620-630.

Whisman, M.A., Perez, J.E., Ramel, W.J. (2000). Factor structure of the Beck Depression Inventory-Second Edition (BDI-II) in a student sample. Journal of Clinical Psychology, 56(4), 545-551.

Wylie, R. C. (1989). Measures of self-concept. Lincoln: University of Nebraska Press.

Zettle, R. D. \& Hayes, S. C. (1986). Dysfunctional control by client verbal behavior: The context of reason giving. The Analysis of Verbal Behavior 4, 30-38.

Zettle, R. D., \& Rains, J. C. (1989). Group cognitive and contextual therapies in treatment of depression. Journal of Clinical Psychology, 45, 438-445.

\section{Author Contact Information:}

Marchion Hinton (first author)

Western Michigan University

Department of Psychology

1903 W. Michigan Ave. 
Kalamazoo, MI 49008-5439

Marchion.j.hinton@wmich.edu

269-387-4497 (phone)

269-387-4550 (fax)

Scott T. Gaynor, Ph.D. (corresponding author)

Western Michigan University

Department of Psychology

1903 W. Michigan Ave.

Kalamazoo, MI 49008-5439

Scott.gaynor@wmich.edu

269-387-4482 (phone)

269-387-4550 (fax)

\section{Behavior Analyst Online Is Looking For Financial Support!}

The Behavior Analyst Online organization is seeking donors to support its cause.

By contributing to the cost of the journals, you will help to keep our journals free. We plan to list our donors (if they desire) on the BAO site.

The categories of donors are:

Champion - \$500.00, Elite - \$250.00, Fellow - \$150.00, Friend - \$50.00

If you would like to contribute, please contact Halina Dziewolska at halinadz@hotmail.com. Please make check payable to Halina Dziewolska, site funder raiser and send the check to 535 Queen Street, Philadelphia, Pa. 19147 